\title{
Sensory Modulation optimises performance of clinicians
}

\author{
Tawanda Machingura, Chris Lloyd
}

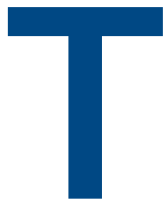

he purpose of this editorial is to explore, through a brief exploration of the current literature and self-reflection, how sensory modulation can be used by clinicians on themselves to optimise their own occupational performance with benefits for both clinicians and ultimately their patients.

We argue that sensory modulation can optimise one's occupational performance and ultimately lead to better outcomes for patients. We recommend that studies be carried out to provide the empirical evidence of this underutilised intervention to improve performance of clinicians and indeed any other workers.

\section{SENSORY MODULATION AND OCCUPATIONAL PERFORMANCE}

Human beings are sensory beings because sensory input is necessary for optimal human brain functioning. The process of regulating one's sensory systems by either calming oneself or alerting oneself to optimise one's performance in everyday activities or in one's life roles is often referred to as sensory modulation. Sensory modulation is defined more formally in the literature as 'neurological function that regulates the body's sensory systems and ability to process incoming stimulus' (Ayres, 1972). Typical sensory modulation enables an individual to self-regulate and react appropriately to their environment, which optimises their occupational performance (Ayers, 1972). There is growing literature that proposes that sensory interventions are effective (Lloyd et al, 2014; Machingura et al, 2017). It is therefore surprising that clinicians are not taking advantage of this and yet they are the proponents of this intervention for their patients.

Clinicians perform complex activities and are required to solve complex problems and make complex decisions. For clinicians to achieve optimal occupational performance they need to have the ability to effectively and efficiently partake in clinical tasks and activities, and this is influenced by their objective physical and mental health capacities, as well as their subjective lived experience (Kielhofner, 2008). Thus, they need to function optimally from the moment they step into the workplace.
Higher order functions, such as reasoning ability and ability to solve problems, is related to efficient somatosensory processing and optimal occupational performance. At a central nervous system level, the cortical centres receive sensory information only after it has been filtered and organised by lower levels such as the brainstem and thalamus. This implies that efficiency at the brainstem and thalamus level can enhance higher order functioning and output actions and be the building blocks of cognitive functioning among other skills. These are the very skills that determine whether we can optimally perform a task, activity, occupation or role or not.

When a clinician — or indeed any person — is under stress, then atypical sensory modulation can occur leading to decreased occupational performance. A person who is experiencing difficulties with filtering sensory input, or has sensory sensitivities, fluctuations in arousal and emotional reactivity cannot be expected to perform at their optimal level (Champagne et al, 2010). In such cases, self-soothing can be used to achieve an optimal arousal state that allows the person to establish emotional stability and enhance occupational performance (Ayres, 1972).

\section{USE OF SELF-REGULATION AND SENSORY DIETS}

We self-regulate from the time we wake up to the time we go to bed. Occupational therapists have use the term 'sensory diet' to describe this process of self-regulation using sensory modulation as part of our daily occupational patterns or routines. We do this by altering our level of arousal. For example, the first author's sensory diet looks something like this: he normally starts his day with a cup of coffee as this gets him going (alerting), then he goes for a quick run on the treadmill in the gym (alerting) and this helps him to be grounded, focused and ready to engage in his work tasks (occupation). Throughout his work day, he finds that his level of arousal fluctuates. Following a well-deserved lunch, he needs to alert himself again and he does this by going for a 20-minute walk during the lunch break. This action prepares him well for the afternoon.

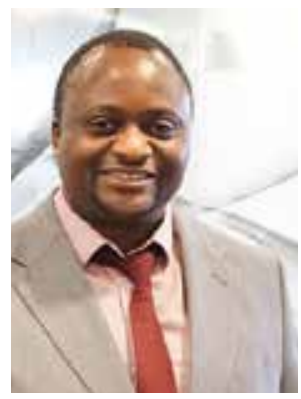

Tawanda Machingura Assistant Professor Occupational Therapy. Bond University, Gold Coast, Queensland; PhD candidate, Griffith University, Queensland, Australia

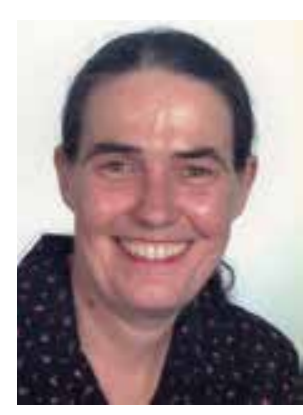

Tawanda Machingura Adjunct Senior Research Fellow, Griffith University, Queensland, Australia 


\section{CONCLUSIONS}

We argue that carefully selected and organised sensations can facilitate performance and hence suggest that clinicians could utilise sensory interventions to optimise their performance. A grounded, focused, alert clinician is less likely to make mistakes and more likely to be able to engage with his or her patients.

We argue that if clinicians feel confident to recommend sensory interventions to their patients, then there is no reason why they should not be using these interventions on themselves. Doing so would be good role modelling and would improve clinicians' knowledge, skills and confidence when recommending this intervention to their patients. Research into the impact of sensory interventions on occupational performance is however still limited and we suggest that researchers in the field of occupational therapy could lead in this area of research. IJTR

Ayres AJ. Types of sensory integrative dysfunction among disabled learners. Am J Occup Ther. 1972;26(1): 13-18.

Champagne T, Koomar J, Olson L. Sensory processing evaluation and intervention in mental health. OT Pract. 2010;15(5):CE1-CE7.

Kielhofner G. Model of Human Occupation Theory and Application. Fourth edition. Baltimore, MD: Lippincott Williams \& Wilkins; 2008

Lloyd C, King R, Machingura T. An investigation into the effectiveness of sensory modulation in reducing seclusion within an acute mental health unit. Adv Ment Health. 2014;12(2): 93-100.

Machingura T, Shum D, Molineux M, Lloyd C. Effectiveness of sensory modulation in treating sensory modulation disorders in adults with schizophrenia: a systematic literature review. Int J Ment Health Addict 2017:1-17. http://dx.doi.org/10.1007/ s11469-017-9807-2 